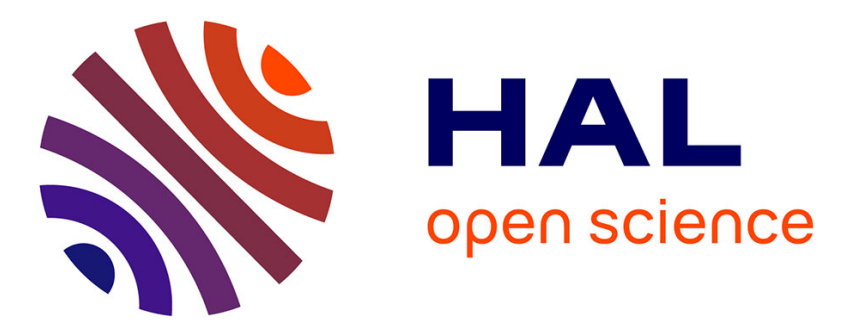

\title{
Nanometric Protein-Patch Arrays on Glass and Polydimethylsiloxane for Cell Adhesion Studies
}

Fuwei Pi, Pierre Dillard, Laurent Limozin, Anne Charrier, Kheya Sengupta

\section{To cite this version:}

Fuwei Pi, Pierre Dillard, Laurent Limozin, Anne Charrier, Kheya Sengupta. Nanometric Protein-

Patch Arrays on Glass and Polydimethylsiloxane for Cell Adhesion Studies. Nano Letters, 2013, 13 (7), pp.3372 - 3378. 10.1021/nl401696m . hal-01780243

\section{HAL Id: hal-01780243 \\ https://hal-amu.archives-ouvertes.fr/hal-01780243}

Submitted on 27 Apr 2018

HAL is a multi-disciplinary open access archive for the deposit and dissemination of scientific research documents, whether they are published or not. The documents may come from teaching and research institutions in France or abroad, or from public or private research centers.
L'archive ouverte pluridisciplinaire HAL, est destinée au dépôt et à la diffusion de documents scientifiques de niveau recherche, publiés ou non, émanant des établissements d'enseignement et de recherche français ou étrangers, des laboratoires publics ou privés. 


\title{
A Versatile Approach to Fabrication of Protein Nano-dot Arrays on Soft Substrates
}

\author{
Fuwei Pi, Pierre Dillard, ${ }^{\dagger}$ Laurent Limozin, Anne Charrier, Kheya Sengupta ${ }^{*}+$
}

†Aix-Marseille Université, CNRS, CINaM UMR 7325, 13288 Marseille, France

† Lab. Adhesion \& Inflammation, Aix-Marseille Université, CNRS UMR 7333, INSERM UMR

1067 , 13288 Marseille, France

* To whom all correspondence should be addressed.

E-mail: sengputa@cinam.univ-mrs.fr 


\begin{abstract}
We present a cost effective simple colloidal bead self-assembly based bench-top protocol for fabricating protein nano-dot arrays on hard and soft substrates. An improved protocol for creating evaporation masks by self-assembly of micro-beads is developed. The masks are subsequently used to create regularly spaced protein dots of variable size, down to about $80 \mathrm{~nm}$, and covering macroscopic areas on glass. The various steps of the fabrication process are characterized by atomic force and epi-fluorescence microscopy. The glass supports can be either used as is for advanced optical microscopic studies (including surface sensitive techniques like total internal reflection microscopy and reflection interference microscopy) of living cells, or can be used as template for further functionalization of soft substrates by contact printing. Glass supported polydimethylsiloxane (PDMS) thin layers, with stiffness ranging from $30 \mathrm{KPa}$ to $3 \mathrm{MPa}$ were functionalized with fluorescent proteins and imaged with an optical microscope.
\end{abstract}

Keywords: Nano-bio-functionalization, Particle Lithography, Adhesion of T-lymphocytes, Soft substrate, Surface sensitive imaging 
Cells have the ability to sense material elasticity, ${ }^{1-3}$ and they respond to the nano-scale organization of active biomolecules ${ }^{4,5}:$ these were two key discoveries in the last couple of decades in the context of cellular interaction with their environment. By varying the stiffness of polymer substrates, it has been shown that depending on the cell type, cells respond to the stiffness of the substrates they adhere to by change in their morphologies, ${ }^{3}$ stiffness,,${ }^{3,6}$ signalling ${ }^{7}$ and differentiation. ${ }^{8}$ Independently, it was shown that on planar glass surfaces, not only the absolute densities of bioactive molecules but even the specific pattern of distribution strongly influences cell behavior. ${ }^{910}$ Bringing together these two aspects in a nano-patterned substrate of controlled elasticity, patterned with regularly spaced protein nano-dots is expected to be an important technological advance towards realizing a platform for probing and manipulating cellular response to the physical and chemical cues in their immediate environment.

In the context of nano-biotechnology a lot of advances have been made in production of biocompatible nano-particles or surfaces exhibiting nano-topography, but techniques for biochemical patterning of surfaces with nano-dots of bioactive molecules remain restricted. One of the most flexible methods in terms of choice of molecules and surfaces is dip pen nanolithography, ${ }^{11}$ but even with massive parallelization, ${ }^{12,13}$ due to the requirement of large coverage for cell biology studies, this remains too slow for regular use in a biology laboratory. Techniques based on contact printing, have been limited mainly by the material properties of the stamp that limit the smallest feature size. ${ }^{14}$ Two alternative approaches - one based on colloidal gold precipitated inside block co-polymer micelles and the other on nano-imprint lithography followed by etching and gold-paladium evaporation, have been successfully used in cell adhesion studies. ${ }^{4,5,10,15,16}$ However, both these approaches rely on selective functionalization of pre-formed gold nano-dots and are therefore limited by the bio-chemistry and optical properties of gold. Of 
these approaches, only the block co-polymer micelle approach has been successfully adapted to soft substrates, ${ }^{17,18}$ but again, they rely on the use of gold nano-clusters.

A different promising approach for nano-patterning is the use of self-assembled mask for vapor deposition of organo-aminosilane molecules that are subsequently functionalized with the desired protein. The amino-silane nano-dots offer virtually no interaction with light, at the same time permitting a large range of strategies for subsequent bio-functionalization. The evaporation mask can be made via phase separation of polymers, ${ }^{19}$ from ordered anodic alumina membranes,${ }^{20}$ or via particle lithography ${ }^{21,22}$ (see Ref. ${ }^{23}$ for a detailed review). Of these, particle lithography has seen considerable development in the past few years, including improvements in ordering, ${ }^{24}$ in functionalization, ${ }^{25}$ use of binary mixture, ${ }^{26}$ independent control of dot size and spacing ${ }^{27}$ etc. The two main concerns still remaining are the issue of controlling the ordering over large surfaces to facilitate applications in biology and the nano-dot size, which ideally should be controlled down to single molecule but to this day remains around $200 \mathrm{~nm}$ on hard inorganic substrate. Particle lithography has so far never been used for functionalization of soft or flexible substrates.

Here we present a versatile and facile technique for large-scale coverage of glass or a soft polymer with ordered protein nano-dots. A novel protocol for producing an evaporation mask by colloidal bead self-assembly is developed. The mask, assembled on glass, serves to successively deposit (a) molecules of organo-amino-silane in the form of a cushion with regularly spaced holes, (b) reactive polyetheleneglycol (PEG) molecules that bind to the amine group, and (c) the protein of interest that binds wherever PEG is not present. On removal of the mask, the slides can be used as is, for example for cell adhesion studies as reported here, or they can be used as 
"masters" for functionalization of soft substrates. For this, the nano-dots of protein are transferred by contact printing to the target soft substrates comprising glass supported planar Polydimethylsiloxane (PDMS) cushions. The stiffness of the PDMS layer is varied by varying the base to cross-linker concentration in the range of $30 \mathrm{KPa}$ to $3 \mathrm{MPa}$.

Fig. 1(a) illustrates the procedure for obtaining large area coverage of a monolayer of colloidal microspheres. In brief, a controlled volume of polystyrene or silica microsphere suspension of a controlled concentration is allowed to spread under gravity on a slightly inclined glass cover-slip, which is pre-cleaned to render it highly hydrophilic. Two simple improvements facilitate uniform coverage: first, the inclination angle is slowly decreased and second, a backflow of the drained bead suspension is effectuated so that the defects in the monolayer formed during the first spreading step are filled over. Using such improved strategy, large scale patterns (up to about $24{ }_{2}^{3} 24 \mathrm{~mm}$ - corresponding to full coverage of the coverslide) of microspheres could be easily obtained without special control of temperature, humidity or other environmental conditions. The important factors that control the quality of the formed monolayers in terms of uniformity and the area covered are: hydrophilicity of the glass surface, the concentration and volume of microsphere suspension. The microsphere size was varied from $500 \mathrm{~nm}$ to $6 \ngtr n$. The overall strategy is the same for both polystyrene and silica microspheres and for the different sizes. The main parameters to be optimized are the concentration and volume used (See Supporting Information for details).

The colloidal bead monolayer was used as a mask to deposit organo-amino-silane molecules on a glass coverslip from the vapor phase. We could successfully graft APTES ((3Aminopropyl)triethoxysilane) and OTS (Octadecyltrichlorosilane), the former being more suitable for subsequent functionalization. The success of the silanization process was tested by 
measuring the contact angle of a droplet of water to verify that the coverslip was rendered hydrophobic.

To characterize the grafting of APTES at the molecular scale, we imaged the surface of the coverslip, after removal of the colloidal bead mask, with atomic force microscopy (AFM). For further discussion here we describe the patterns fabricated with polystyrene microspheres with 1 $¥$ diameter as representative. We have verified that the results are similar for other bead sizes and for silica beads. Fig. 2a shows AFM image after the APTES deposition stage. An array of circles is seen. The spacing of $980{ }_{93}^{238} 10 \mathrm{~nm}$ corresponds well to the diameter of the beads used (Fig. 2b; see also Table 1a for all statistics). The inner radius of the circles is $120{ }_{93}^{232} 20 \mathrm{~nm}$. The height profile across a single circle demonstrates that the inside of the circle either remains bare or is very sparsely grafted with APTES (Fig. 2c and d). The height difference of $1.5{ }_{93}^{23} 0.2 \mathrm{~nm}$ (statistics from $>10$ motifs) between the inside and the outside corresponds well to the expected size of an APTES molecule. We conclude that the APTES molecules are grafted on the glass surface in the form of a monolayer, but in the confined space between the bead and the substrate they form multilayers.

At this stage, the substrate is patterned with patches of bare glass surrounded by a sea of APTES molecules. To proceed further with the functionalization, PEG chains were grafted on to APTES by submerging the silanized glass coverslip into an aqueous solution of NHS-PEG ester (Fig. 1b). An alternative strategy was to graft PEG-COOH via peptide bonds in an alkaline medium overnight (see Supporting Information for details). While the first strategy is quick and facile, the second offers more flexibility in terms of availability of different kinds of PEG 
molecules. In both cases, the quality of the pattern is better if the colloidal bead mask is removed after, rather than before, grafting the PEG molecules. The success of the PEG binding step could be easily verified by measuring the contact angle of a droplet of water, showing that the slides become hydrophilic again after this step. At this stage, the glass coverslip is patterned with submicron holes of bare glass in a sea of PEG. In the subsequent step, the bare glass holes are filled with a desired protein, with the PEG molecules acting as repellers to prevent protein deposition elsewhere. Different chain lengths of PEG were tested and the one most suitable for the subsequent protein binding step, namely 36 units, was retained.

The coverslips with patterned PEG were functionalisatized with the protein of interest by incubating in a suitable solution of the protein molecule for a controlled amount of time (Fig. 1c). The protein molecules are expected to adsorb only on the patches of bare glass corresponding to the contact sites of microspheres in the original mask, thus forming a regular protein island array corresponding to the original pattern of the microspheres. Typically, fluorescent bovine serum albumin (BSA) was used for establishing the protocol. The robustness of the protocol was then tested using BSA-biotin which was subsequently functionalized with the avidin analogue fluorescent neutravidin.

The fluorescent protein islands were imaged with epi-fluorescence (Fig. 3) or atomic force (Fig. 4) microscopy. Fig. 3 shows a few typical examples of the patterns formed with different parameters for the bead-mask and different choice of proteins. The pitch corresponds to the diameter of the beads used to fabricate the mask. Comparison of AFM and optical imaging data for bead sizes of $500 \mathrm{~nm}, 1 \mp$ or $2 ¥$ diameter (Table 1a), shows that for both the silica and the polystyrene beads, the size of the protein nano-dots measured in epi-fluorescence from the 
full width at half maximum of the intensity trace, is larger than the size measured in AFM. This is probably because the optical images are resolution limited. Fig. $3 \mathrm{~g}$ and $3 \mathrm{~h}$ are of particular interest - they illustrate the possibility of changing the patch size without changing the inter-patch distance ${ }^{28}$. To achieve this, the colloidal bead mask, prepared from $6 \ngtr$ PS beads, is gently heated after its formation and just prior to APTES deposition. This melts the polymer and increases the foot-print of the bead on the glass surface thus giving rise to an expanded patch size.

AFM image of BSA protein patches (fabricated with $1 \neq n$ diameter microspheres), is presented in Fig. 4a. This can be compared with Fig. 3a where epi-fluorescence images (fluo) of the same substrate is presented. The measured pitch of $1000{ }_{93}^{238} 30 \mathrm{~nm}$ (fluo) and $960{ }_{93}^{238} 65 \mathrm{~nm}$ (AFM) correspond closely to the pitch of APTES circle arrays imaged in Fig. 2, as well as to the diameter of the colloidal beads used to fabricate the mask. The patch size measured in fluorescence images $(390 \pm 20 \mathrm{~nm})$ is larger than the true size $(115 \pm 20 \mathrm{~nm})$ measured from AFM. The concentration of the solution from which the protein is deposited regulates the amount of protein in the patches, as measured from the height of the protein patch with respect to the surrounding layer of PEG (data not shown). When deposited from $10 \neq / \mathrm{ml} \mathrm{BSA/PBS} \mathrm{solution,}$ the height difference is about $3 \mathrm{~nm}$ (Fig. 4d). Comparing Fig. 4 with Fig. 2, it is seen that whereas in Fig 2, the interior of the imaged circles is lower than the surrounding sea of APTES, in Fig. 4, the interior of the circles is higher than the surrounding sea of PEG. Comparison with epi-fluorescence images clearly indicate that the extra material corresponds to the fluorescent protein. 
In all the cases tested, the pitch of the final protein nano-dot array equals the diameter of the bead type used to fabricate the mask. The nano-dot size however is only weakly dependent on the diameter of the beads used in the mask (Table 1a). The smallest size obtained is for $500 \mathrm{~nm}$ diameter beads and is $80 \mathrm{~nm}$ as measured by AFM.

These protein patterned glass slides were used as support for pilot experiments on $\mathrm{T}$ lymphocyte adhesion. For this, BSA-biotin was patterned as described and was subsequently functionalized with fluorescent streptavidin (visualized in Fig. 5a), which in turn was further functionalized with mono-biotinylated anti-CD3 (OKT3). T-lymphocytes (cell line Jurkat) were allowed to interact with these substrates. It is expected that the sea of PEG provides a passive background with activating anti-CD3 islands at regular intervals. The live cells were monitored with RICM $^{29}$ (Fig 5b). After spreading, the cells were fixed, stained for T-cell receptor (TCR) molecules and imaged in TIRF (Fig 5c) and IRM ${ }^{29}$ (Fig 5d) mode. IRM and RICM images show that the cells adhere not only on the functionalized patches but also on the PEG. This is consistent with control experiments on PEG alone. As expected, the TCR forms micro-clusters on the cell surface ${ }^{30}$ but unexpectedly, the TCR microclusters are distributed randomly, as also seen in control experiments on uniform anti-CD3, rather than co-localizing with the anti-CD3 patches. These pilot experiments confirm that the current organo-amino-silane based functionalization protocol is perfectly suited for advanced optical imaging.

The protein-dot array on glass can also serve as a flat stamp from which the protein nano-dots can be readily transferred to the surface of a soft polymer like PDMS. The process is the inverse of conventional micro-contact printing and is summarized in Fig. 1d. First, the PDMS substrates are prepared by sandwiching non-reticulated PDMS (base mixed with desired amount of cross- 
linkers) between a glass coverslip which serves as support and a smooth passivated glass slide, separated by glass spacers of desired thickness (typically 140 microns). The PDMS is then cured and the passivated slide is removed to yield the substrate which comprises a glass coverslip supporting a thin film of PDMS with very smooth surface. This PDMS surface is then manually put in contact with the previously prepared protein-master. On separation, the protein dots are seen to have been transferred to the PDMS surface. The stiffness of the PDMS can be controlled by controlling the amount of cross-linkers used. An estimated stiffness of $30 \mathrm{KPa}, 140 \mathrm{KPa}$ and 3 MPa was produced by choosing base to cross-linker ratio of 50:1, 35:1 and 10:1 respectively. ${ }^{31,32}$ Below we refer to these as soft, medium and stiff substrates.

Fig. 6 shows representative fluorescence images of protein nano-dots transferred from masters fabricated with $2 ¥ n$ diameter microspheres onto the stiff, medium and soft PDMS substrates. It is apparent that the protein dots on PDMS retain the essential features of the master (corresponding to Fig. 2a) for all three stiffness presented here. The pitch of the transferred protein pattern on the PDMS $\left(1930{ }_{93}^{238} 40 \mathrm{~nm}, 1820{ }_{93}^{238} 40 \mathrm{~nm}\right.$ and $1860{ }_{93}^{238} 0 \mathrm{~nm}$ for stiff, medium and soft respectively) closely corresponds to that of the master $\left(1920{ }_{93}^{238} 30 \mathrm{~nm}\right)$. The lateral size of the dots, corresponding to the full width at half maximum of the intensity line profiles from the images ( $390{ }_{932}^{238} 0 \mathrm{~nm}, 480{ }_{9330}^{238} \mathrm{~nm}$ and $390{ }_{93}^{238} 10 \mathrm{~nm}$ for stiff, medium and soft respectively) is slightly larger than the size of the dots on the master $\left(310{ }_{93}^{238} 30 \mathrm{~nm}\right)$. This probably happens due to deformability of the PDMS substrate as well as the imprecise nature of manual transfer. We have successfully transferred patterns from masters fabricated using both silica and polystyrene beads of different size. The observed patch size and inter patch distance on masters 
at different steps (measured from optical and AFM images) and of the protein nano-dots on PDMS substrates (measured from optical images) are summarized in Table $1 \mathrm{~b}$.

Here we have reported a simple cost-effective strategy for large scale patterning of protein nano-dots on hard and soft substrates. The technique can be easily implemented as a bench top setup, bypassing many of the current limitations on fabricating nanoscale masters, which rely on expensive and specialized facilities and absolutely clean environment. Our approach uses only commercially available reagents and chemicals, thus avoiding complicated chemical synthesis or nano-fabrication required for gold-nano dot based approaches. Since the transfer is from one planar surface to another, many of the problems associated with microcontact printing of very small features are absent. Such functionalized PDMS substrates hold out the promise of mechanosensing studies combined with nano-scale surface stimulation of living cells. We believe that this generic method will lead to a popular route for bench-top patterning of bioactive molecules over a large area on hard as well as soft substrates for exploring the molecular, cellular and biophysical basis of cell behavior at the nanoscale.

Acknowledgments: We thank Loïc Magiera-Millé for pilot experiments on colloidal bead masks, Herve Dallaporta and Frank Thibaudau for fruitful discussions, Anne Marie Lellouch and Sophie Cadra for help with T-cells and Rajat Varma for gift of monobiotinylated OKT3. KS and FP acknowledge funding from region-PACA (grant no. 2011_06554). The research leading to these results has also received funding from the European Research Council under FP/20072013/ ERC Grant Agreement n. 307104. 


\section{References}

(1) Pelham, R. J.; Wang, Y-L. Proc. Natl. Acad. Sci. 1997, 94, 13661-13665.

(2) Discher, D. E.; Janmey, P.; Wang, Y. Science 2005, 310, 1139-1143.

(3) Solon, J.; Levental, I.; Sengupta, K.; Georges, P. C.; Janmey, P. A. Biophys. J. 2007, 93, 4453-4461.

(4) Geiger, B.; Spatz, J. P.; Bershadsky, A. D. Nat. Rev. Mol. Cell Biol. 2009, 10, 2133.

(5) Deeg, J. A. Louban, I.; Aydin, D.; Selhuber-Unkel, C.; Kessler, H.; Spatz, J. P. Nano Lett. 2011, 11, 1469-1476.

(6) Tee, S.; Fu, J.; Chen, C. S.; Janmey, P. A. Biophys. J. 2011, 100, L25-L27.

(7) Levental, K. R.; Yu, H.; Kass, L.; Lakins, J. N.; Egeblad, M.; Erler, J. T.; Fong, S.; Csiszar, K.; Giaccia, A.; Weninger, W.; Yamauchi, M.; Gasser, D. L. Weaver, V. M. Cell 2009, 139, 891-906.

(8) Engler, A. J.; Sen, S.; Sweeney, H. L.; Discher, D. E. Cell 2006, 126, 677-689.

(9) Huang, J.; Grater, S. V.; Corbellini, F.; Rinck, S.; Bock, E.; Kemkemer, R.; Kessler, H.; Ding, J.; Spatz, J. P. Nano Lett. 2009, 9, 1111-1116.

(10) Schvartzman, M.; Palma, M.; Sable, J.; Abramson, J.; Hu, X.; Sheetz, M. P.; Wind, S. J. Nano Lett. 2011, 11, 1306-1312.

(11) Piner, R. D.; Zhu, J.; Xu, F.; Hong, S.; Mirkin, C. A. Science 1999, 283, 661663. 
(12) Lee, K.; Park, S.; Mirkin, C. A.; Smith, J. C.; Mrksich, M. Science 2002, 295, 1702-1705.

(13) Salaita, K.; Wang, Y.; Mirkin, C. A. Nat. Nanotechnol. 2007, 2, 145-155

(14) Xia, Y.; Rogers, J. A.; Paul, K. E.; Whitesides, G. M. Chem. Rev. 1999, 99, $1823-$ 1848.

(15) Cavalcanti-Adam, E. A.; Volberg, T.; Micoulet, A.; Kessler, H.; Geiger, B.; Spatz, J. P. Biophys. J. 2007, 92, 2964-74.

(16) Lohmüller, T.; Triffo, S.; O’Donoghue, G. P.; Xu, Q.; Coyle, M. P.; Groves, J. T. Nano Lett. 2011, 11, 4912-4918.

(17) Graeter, S. V.; Huang, J.; Perschmann, N.; López-García, M.; Kessler, H.; Ding, J.; Spatz, J. P. Nano Lett. 2007, 7, 1413-1418.

(18) Aydin, D.; Louban, I.; Perschmann, N.; Blummel, J.; Lohmuller, T.; CavalcantiAdam, E. A.; Haas, T. L.; Walczak, H.; Kessler, H.; Fiammengo, R.; Spatz, J. P. Langmuir 2010, 26, 15472-15480.

(19) Vogelaar, L.; Barsema, J. N.; Nijdam, W.; van Rijn, C. J. M.; Wssling, M. Adv. Mater. 2003, 15, 1385-1389.

(20) Massou, S.; Masson, L.; Ozerov, I.; Moyen, E.; Sengupta, K.; Hanbücken, M. Appl. Surf. Sci. 2009, 256, 395-398.

(21) Li, J. R.; Garno, J. C. Nano Lett. 2008, 8, 1916-1922.

(22) Zhang, J.; Li, Y.; Zhang, X.; Yang, B. Adv. Mater. 2010, 22, 4249-4269. 
(23) Lei, Y.; Yang, S.; Wu, M.; Wilde, G. Chem. Soc. Rev., 2011, 40, 1247-1258.

(24) Colson, P.; Cloots, R.; Henrist, C. Langmuir 2011, 27, 12800-12806.

(25) Wolf, C.; Qin, L. Langmuir 2010 26, 11605-12472.

(26) Singh, G.; Pillai, S.; Arpanaei, A.; Kingshott, P Adv. Mater. 2011 24, 1519-1523.

(27) Mukhopadhyay, R.; Al-Hanbali, O.; Pillai, S.; Hemmersam, A. G.; Meyer, R. L.; Hunter, A. C.; Rutt, K. J.; Besenbacher, F.; Moghimi, S. M.; Kingshott, P. J. Am. Chem. Soc. 2007, 129, 13390-13391.

(28) Taylor, Z. R.; Keay, J. C.; Sanchez, E. S.; Johnson, M. B.; Schmidtke, D. W. Langmuir 2012, 28, 9656-9663.

(29) Limozin, L.; Sengupta, K. ChemPhysChem 2009, 10, 2752-2768.

(30) a. Kaizuka, Y.; Douglass, A.; Varma, R.; Dustin, M.; Vale, R.D. Proc. Natl. Acad. Sci. 2007, 104, 20296-20301, b. Seminario, M. C.; Bunnell S. C. Immunol Rev. 2008, 221, 90-106.

(31) Ceșa, C. M.; Kirchgeßner, N.; Mayer, D.; Schwarz, U. S.; Hoffmann, B.; Merkel, R. Rev. Sci. Instrum. 2007, 78, 034301- 034310.

(32) Merkel, R.; Kirchgeßner, N.; Ceșa, C. M. Hoffmann, B. Biophys. J. 2007, 93: 3314-3323. 
Table 1a. The size of APTES and protein nano-dots on glass as measured by AFM or epifluorescence (fluo)*.

\begin{tabular}{|c|c|c|c|}
\hline $\begin{array}{c}\text { Bead } \\
\text { Size/Type }\end{array}$ & $\begin{array}{c}\text { APTES (AFM) } \\
(\mathrm{nm})\end{array}$ & $\begin{array}{c}\text { Protein (AFM) } \\
(\mathrm{nm})\end{array}$ & $\begin{array}{c}\text { Protein (fluo) } \\
\text { (nm) }\end{array}$ \\
\hline $\begin{array}{c}6 \nsucceq n \\
\text { Polystyrene }\end{array}$ & $\begin{array}{l}3 \\
2\end{array}$ & $\begin{array}{l}3 \\
2\end{array}$ & $750 \quad{ }_{93}^{23} 60$ \\
\hline$m \neq n$ Silica & $190 \underset{93}{238}$ & $150 \pm 15 \mathrm{~nm}$ & $310 \pm 30$ \\
\hline $\begin{array}{c}\qquad \dot{M} \neq n \\
\text { Polystyrene }\end{array}$ & $120{ }_{93}^{238} 10$ & $120 \pm 20$ & $390 \pm 20$ \\
\hline ṕî́ nm Silica & $60{ }_{93}^{238} 10$ & $80 \pm 10$ & $240 \pm 10$ \\
\hline
\end{tabular}

Table 1b. The size of protein nano-dots (fabricated with $m ¥ n$ Silica beads as mask) on PDMS substrates measured by epi-fluorescence *.

\begin{tabular}{lccc}
\hline \multirow{2}{*}{ Glass } & \multicolumn{3}{c}{ PDMS } \\
\cline { 2 - 4 } $310 \pm 30 \mathrm{~nm}$ & $10: 1(3 \mathrm{MPa})$ & $35: 1(140 \mathrm{KPa})$ & $50: 1(30 \mathrm{KPa})$ \\
\cline { 2 - 4 } & $390 \pm 10 \mathrm{~nm}$ & $480 \pm 30 \mathrm{~nm}$ & $390 \pm 20 \mathrm{~nm}$
\end{tabular}

${ }^{\circ} \mathrm{FAFM}$ data are the averaged values of at least 10 motifs; Fluorescence data, as measured from FWHM of intensity profiles are for at least 10 dots for 3 samples. ${ }_{2}^{3}$ indicates data not available. Error bars correspond to standard deviation.

\section{Figure Captions}

Figure 1. Schematic representation of the fabrication strategy for developing large-scale protein nanopatterns on soft substrates (a) self-assembly of the colloidal bead mask on glass substrate, (b) deposition of APTES from vapor phase through the mask and functionalizing with PEG molecules, (c) functionalization with protein, and (d) transfer of the protein nano-dots onto soft, glass supported PDMS. The pitch "d", as measured from AFM and fluorescence imaging is equal to the diameter of the beads used to fabricate the masks. The patch size " $\omega$ " depends weakly on the bead diameter and could be varied from 80 to $200 \mathrm{~nm}$. 
Figure 2. (a) Tapping mode AFM image of glass after grafting APTES (mask: 1 ¥n diameter beads ); (b) corresponding height profile of the line drawn in (a); (c) zoom-in 3D image of a single circle; (d) corresponding profile. Red dash line indicates the height of APTES molecule grafted on the surface, which is $1.5+/-0.2 \mathrm{~nm}$ as measured from $>10$ motifs and is consistent with the grafting of a monolayer of APTES.

Figure 3. Epi-fluorescence images of protein patch arrays fabricated with different types of masks. (a) fluorescentBSA (mask: $2 ¥$ silica beads); (b) fluorescent-BSA (mask: $1 ¥ n$ PS beads); (c) Neutravidin bound to BSA-biotin (mask: $500 \mathrm{~nm}$ silica beads). (d), (e) and (f) are corresponding plot of the intensity profiles. (g and h): BSA patterns fabricated with $6 \mp n$ PS beads at room temperature -RT (g) or pretreated at $110{ }^{\circ} \mathrm{C}(\mathrm{h})$; (i) corresponding intensity profiles. Insets display the Fourier transforms of the corresponding images to emphasize the ordering of the lattice.

Figure 4. (a) Tapping mode AFM image of protein nano-dots fabricated through depositing $300 ¥$ of $10 \notin / \mathrm{ml}$ BSA/PBS solution, using $2 \Varangle n$ diameter beads for mask; (f) corresponding intensity profile of the line drawn in (e). Red and blue dashed lines indicate the height of PEG grafted on APTES and protein adsorbed on glass respectively. The height difference of about $3 \mathrm{~nm}$ indicates adsorption of multiple layers of proteins on glass.

Figure 5. Spreading of Jurkat cell on functionalized glass substrate (mask from 2 ¥n silica beads) (a) epifluorescence image of neutravidin dots functionalized with anti-CD3 and surrounded by a sea of non-fluorescent PEG; (b) a single Jurkat cell imaged in RICM (c) TIRF-M image of another cell, on the same substrate as a ; (d) corresponding IRM image.

Figure 6. Epi-fluorescence images of protein nano-dot arrays on hard (a), medium (b): and soft (c) PDMS layers. Insets display the Fourier transforms.; (d), (e) and (f) are corresponding intensity profiles of the lines drawn. The pitch and the size (HWFM) are indicated. 
(a) Patterning Microspheres

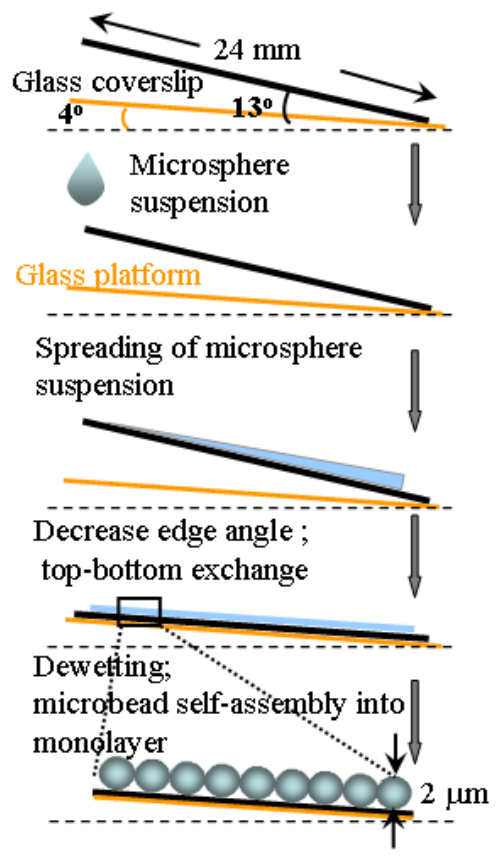

(b) Functionalizing Pattern
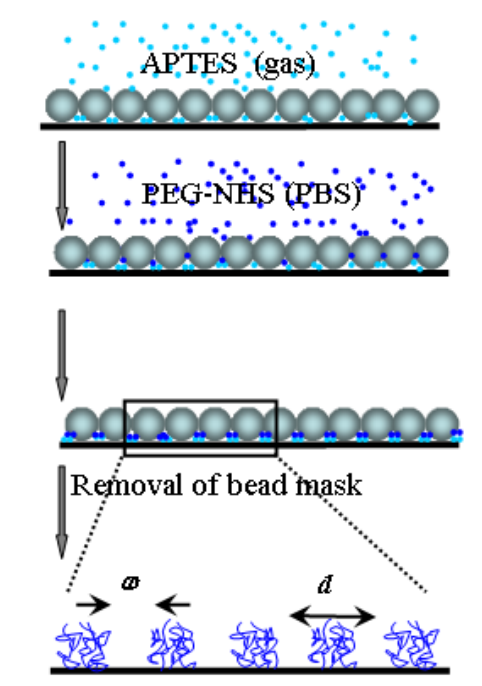

Note: Nanodot size $(\omega)=80$ to $200 \mathrm{~nm}$

Pitch $(d)=0.5$ to $6 \mu \mathrm{m}$ (c) Functionalisation with protein

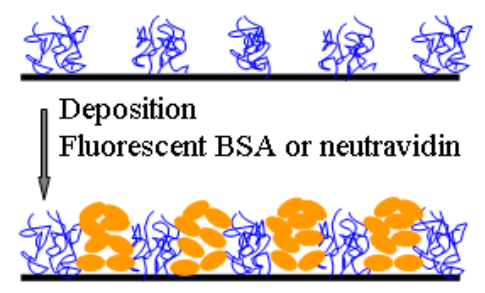

(d) Transfer nanopattern to PDMS

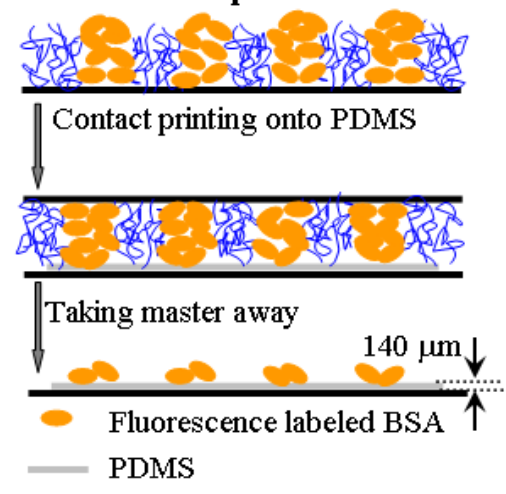

Figure 1. Schematic representation of the fabrication strategy for developing large-scale protein nanopatterns on soft substrates (a) self-assembly of the colloidal bead mask on glass substrate, (b) deposition of APTES from vapor phase through the mask and functionalizing with PEG molecules, (c) functionalization with protein, and (d) transfer of the protein nano-dots onto soft, glass supported PDMS. The pitch "d", as measured from AFM and fluorescence imaging is equal to the diameter of the beads used to fabricate the masks. The patch size " $\omega$ " depends weakly on the bead diameter and could be varied from 80 to $200 \mathrm{~nm}$. 

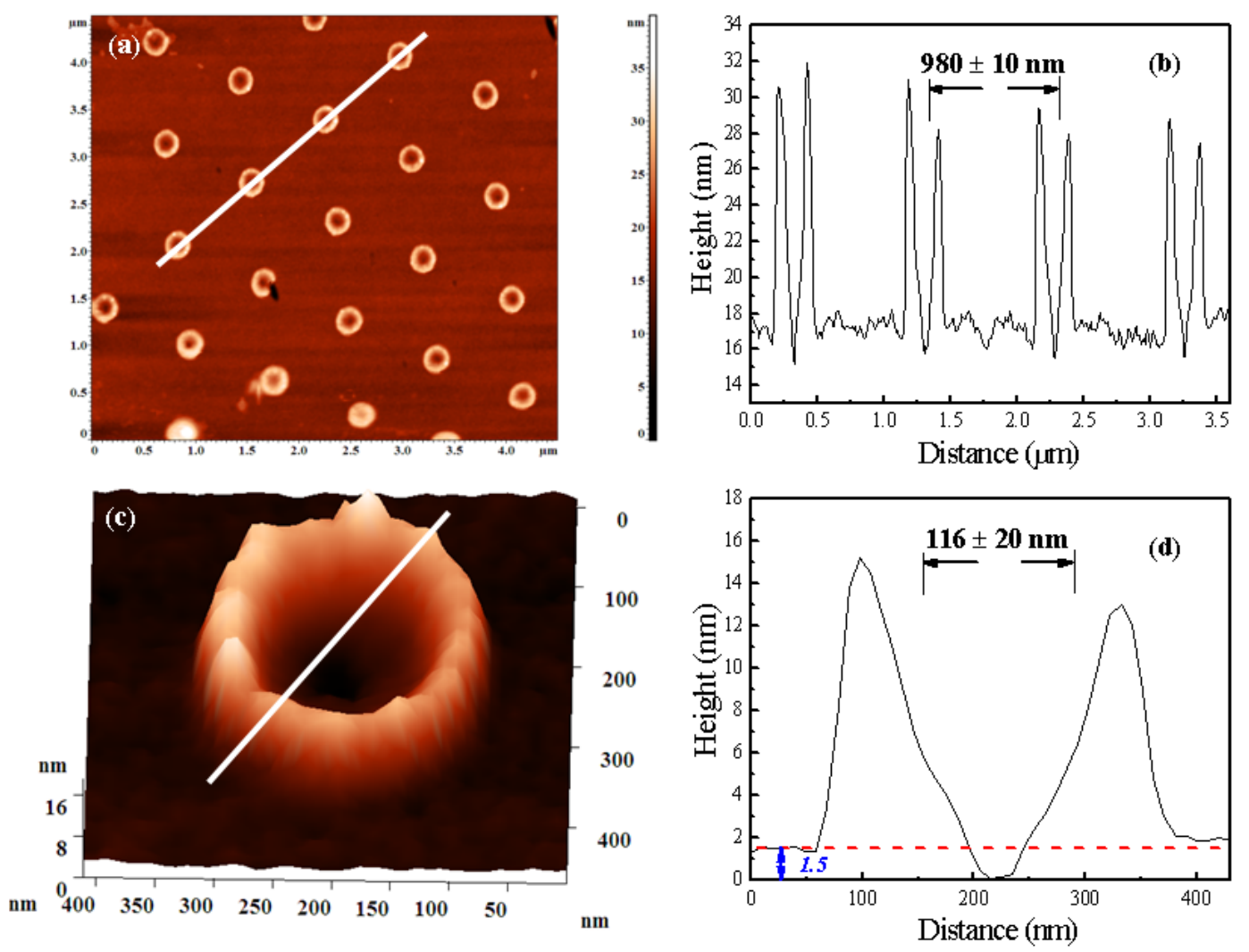

Figure 2. (a) Tapping mode AFM image of glass after grafting APTES (mask: $1 ¥$ diameter beads ); (b) corresponding height profile of the line drawn in (a); (c) zoom-in 3D image of a single circle; (d) corresponding profile. Red dash line indicates the height of APTES molecule grafted on the surface, which is $1.5+/-0.2 \mathrm{~nm}$ as measured from $>10$ motifs and is consistent with the grafting of a monolayer of APTES. 

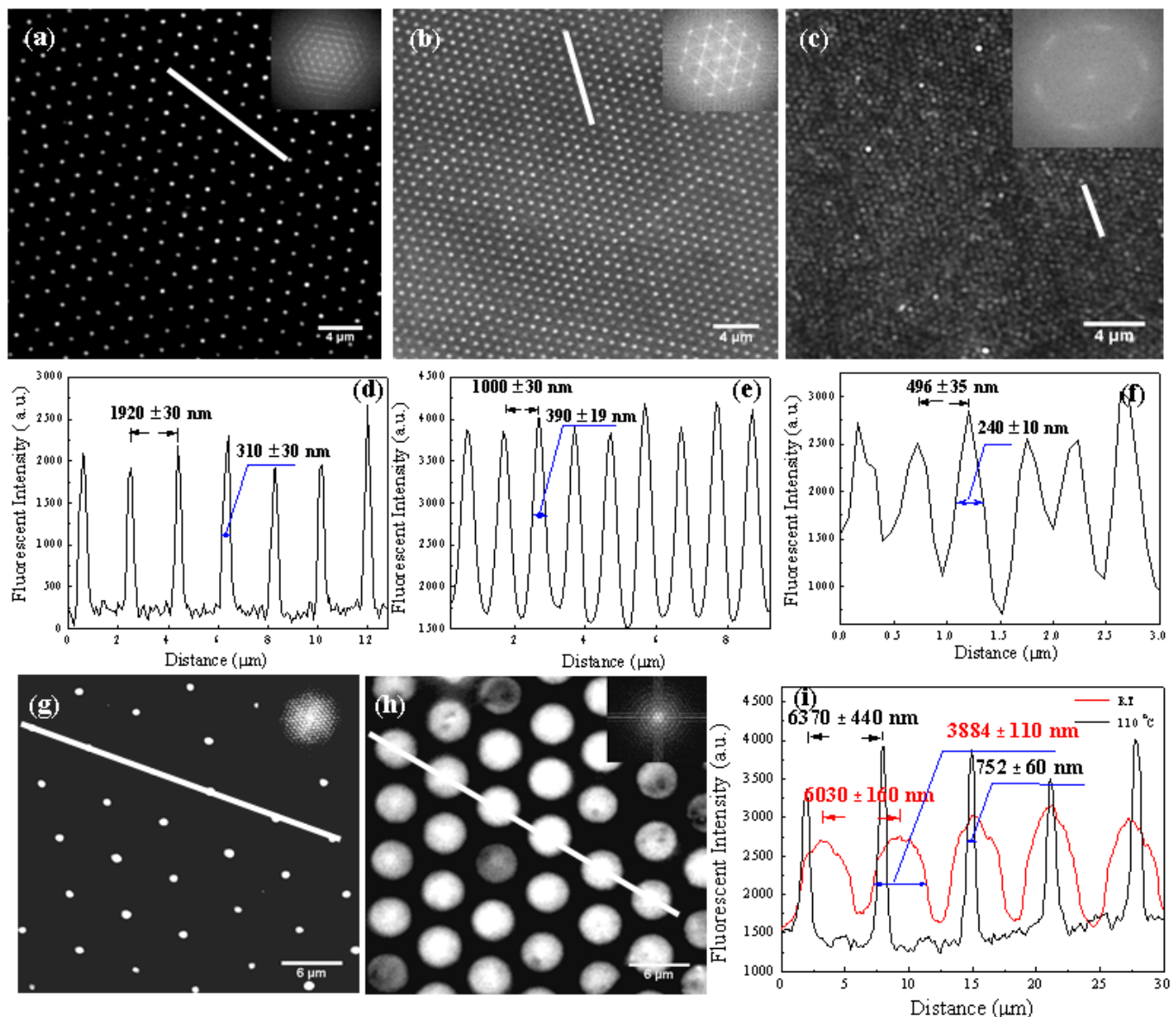

Figure 3. Epi-fluorescence images of protein patch arrays fabricated with different types of masks. (a) fluorescentBSA (mask: $2 ¥ n$ silica beads); (b) fluorescent-BSA (mask: $1 ¥ n$ PS beads); (c) Neutravidin bound to BSA-biotin (mask: $500 \mathrm{~nm}$ silica beads). (d), (e) and (f) are corresponding plot of the intensity profiles. (g and h): BSA patterns fabricated with $6 \neq n$ PS beads at room temperature -RT (g) or pretreated at $110{ }^{\circ} \mathrm{C}(\mathrm{h})$; (i) corresponding intensity profiles. Insets display the Fourier transforms of the corresponding images to emphasize the ordering of the lattice. 

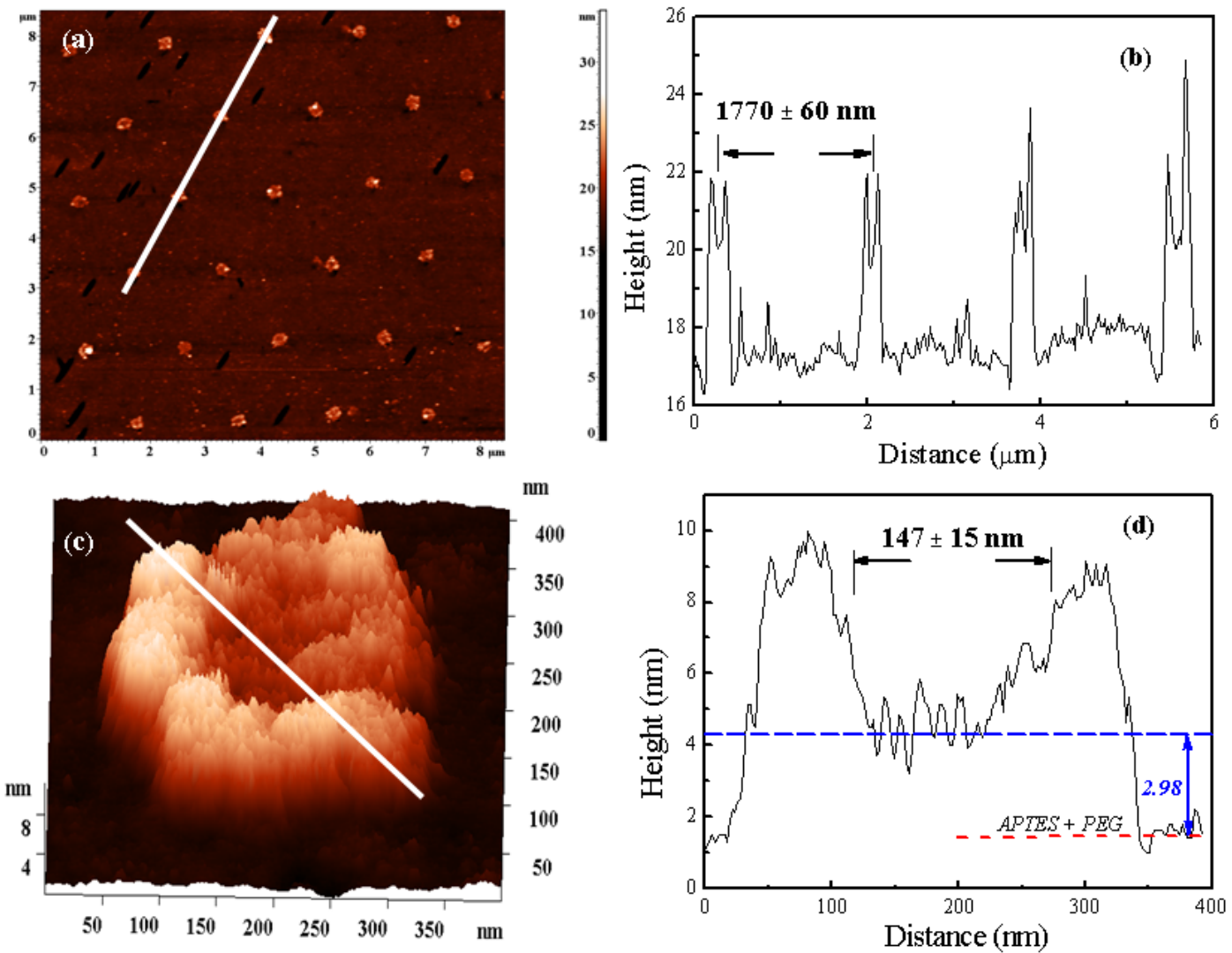

Figure 4. (a) Tapping mode AFM image of protein nano-dots fabricated through depositing $300 \quad$ of $10 \neq / \mathrm{ml}$ BSA/PBS solution, using $2 ¥ n$ diameter beads for mask; (f) corresponding intensity profile of the line drawn in (e). Red and blue dashed lines indicate the height of PEG grafted on APTES and protein adsorbed on glass respectively. The height difference of about $3 \mathrm{~nm}$ indicates adsorption of multiple layers of proteins on glass. 

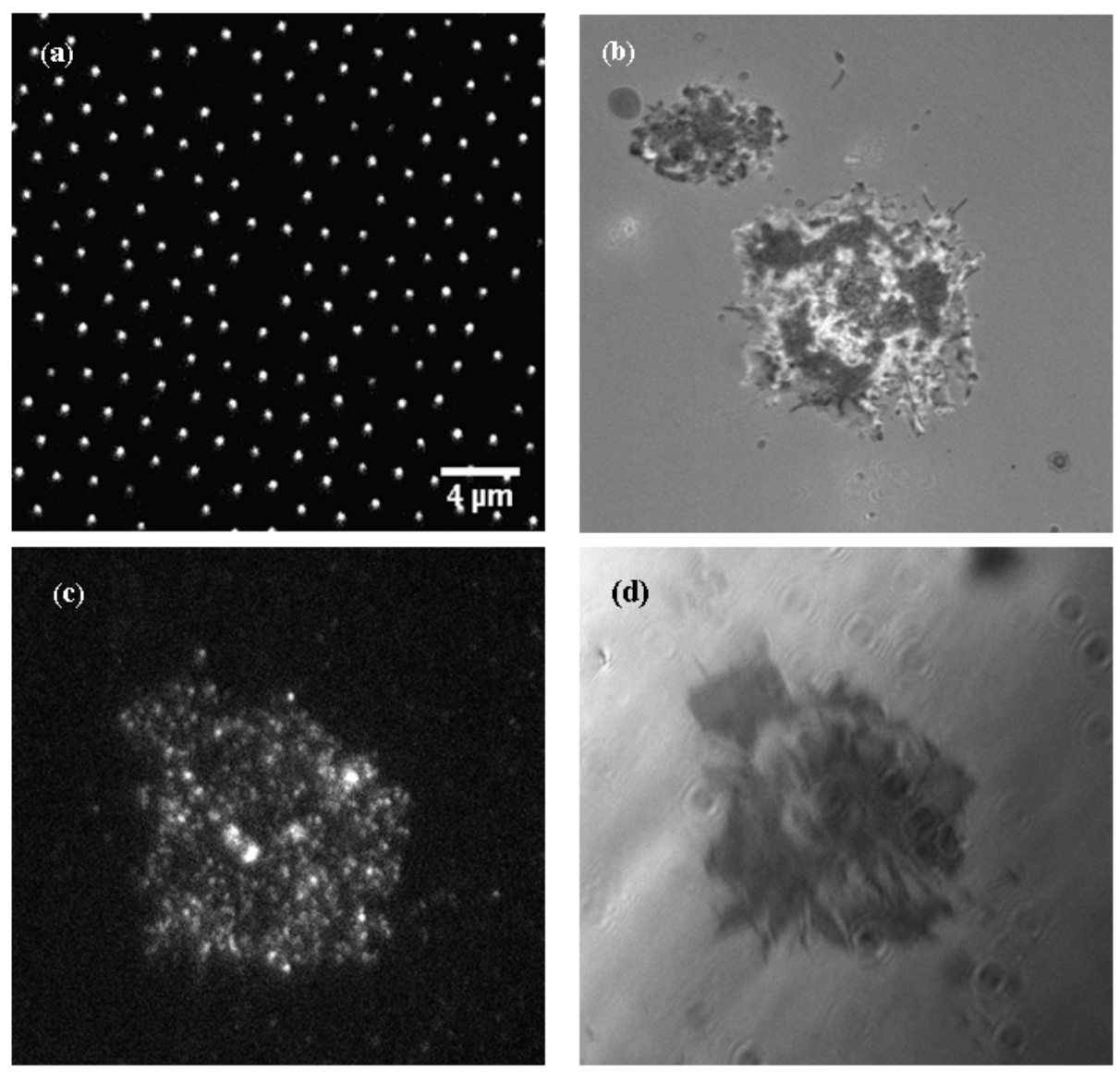

Figure 5. Spreading of Jurkat cell on functionalized glass substrate (mask from 2 ¥n silica beads) (a) epifluorescence image of neutravidin dots functionalized with anti-CD3 and surrounded by a sea of non-fluorescent PEG; (b) a single Jurkat cell imaged in RICM (c) TIRF-M image of another cell, on the same substrate as a ; (d) corresponding IRM image. 

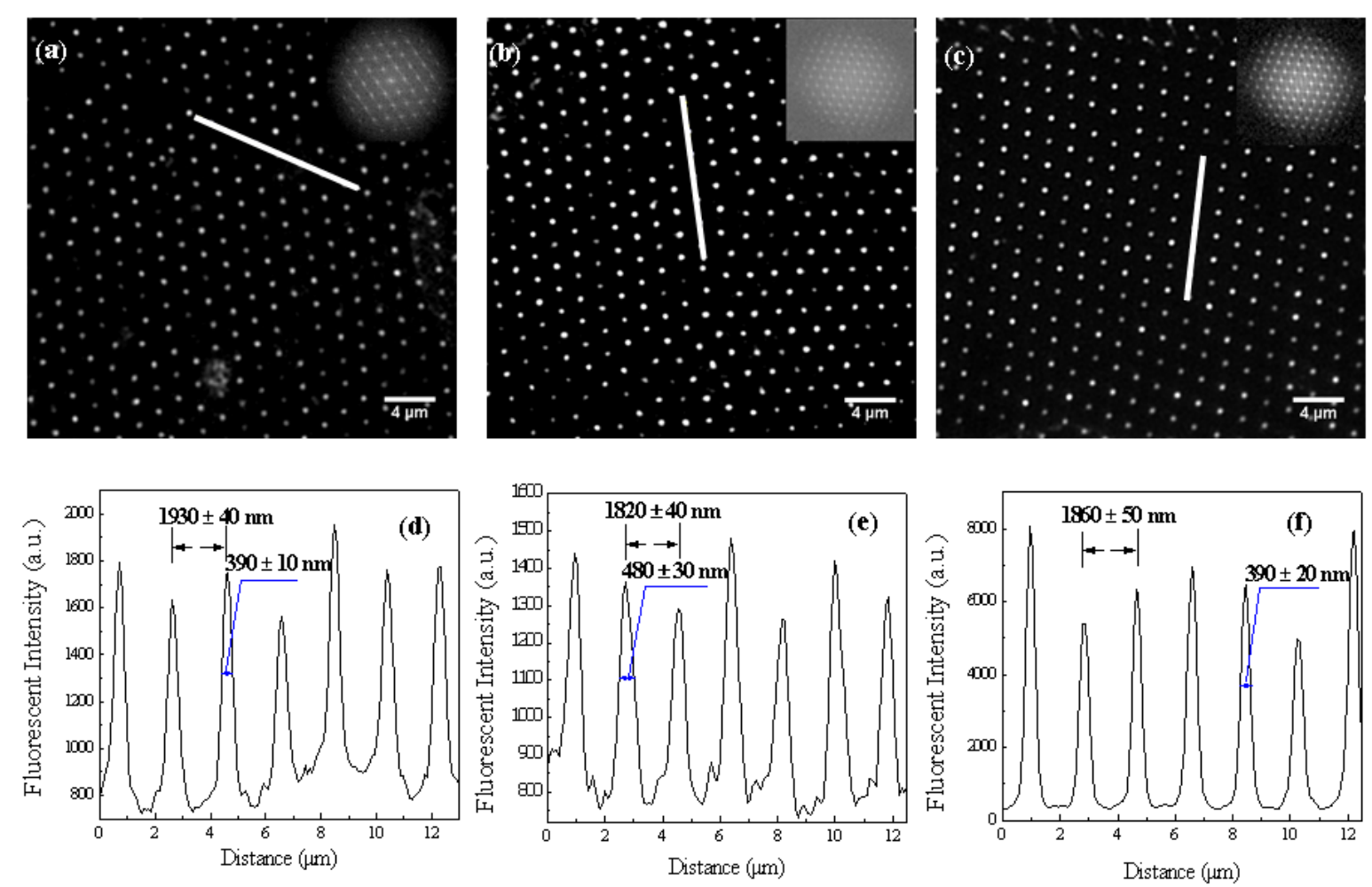

Figure 6. Epi-fluorescence images of protein nano-dot arrays on hard (a), medium (b): and soft (c) PDMS layers. Insets display the Fourier transforms.; (d), (e) and (f) are corresponding intensity profiles of the lines drawn. The pitch and the size (HWFM) are indicated. 


\section{Supporting Information}

\section{A Versatile Approach to Fabrication of Protein Nano-dot Arrays on Soft Substrates}

Fuwei Pi, ${ }^{\dagger}$ Pierre Dillard, ${ }^{\dagger}$ Laurent Limozin, ${ }^{\dagger}$ Anne Charrier, ${ }^{\dagger}$ Kheya Sengupta ${ }^{*}{ }^{\dagger}$

†Aix-Marseille Université, CNRS, CINaM UMR 7325, 13288 Marseille, France

†Lab. Adhesion \& Inflammation, Aix-Marseille Université, CNRS UMR 7333, INSERM UMR 1067 , 13288 Marseille, France 


\section{Experimental Section}

\section{Preparation of glass substrates.}

Glass coverslips (thickness = 170 microns, Assistent, Karl Hecht KG, Germany), $24{ }_{2}^{3} 24$ mm, were cleaned according to the following protocol: ultrasonication in $2 \%$ ( $X \backslash$ aqueous solution of Hellmanex (Sigma, France) for 20 minutes, rinsing 10 times with ultrapure water (resistivity of 18.2 Mø cm, Elga, UK), again ultrasonication in $2 \%$ Hellmanex solution for 20 minutes; ultrasonication in ultrapure water ( 2 times 20 minutes) with repeated rinsing; finally blow-drying with nitrogen stream. Note that this procedure renders the glass ultra hydrophilic (contact angle less than $5^{\circ}$ ). Such high hydrophilicity is essential for the subsequent step of mask formation. Simpler cleaning procedures, for example with organic solvents or piranha solution were found to be insufficient.

\section{Fabrication of colloidal bead masks.}

Suspensions of colloidal microspheres (beads) with different diameters and made either of polystyrene (PS) or of Silica were purchased from Polysciences, Germany and were were washed 
6 times with ultrapure water before utilisation. The following beads were used: of $6 ¥ n$ and $1 ¥ n$ microspheres (PS) concentration $2.53 \%$ and $2.61 \%$ respectively, or $2 ¥ n$ and $500 \mathrm{~nm}$ silica microspheres concentration $10 \%$ and $9.83 \%$ respectively (). The PS colloidal suspensions were used directly; silica colloidal suspensions were diluted into 1:5 and 1:8 ( $X \backslash$ with ultrapure water for $2 ¥ n$ and $500 \mathrm{~nm}$ diameter respectively.

A glass platform with edge angle of $4^{\circ}$ was set up on an ordinary laboratory bench under ambient conditions. Next, a glass coverslip, thoroughly cleaned as described above, was set on the platform at an angle of $13^{\circ}$ such that the colloidal bead suspension spread under gravity. The edge angle of glass coverslip was decreased to $4^{\circ}$ when the spreading front of the suspension reached the bottom of glass coverslip. Uniform spreading was further facilitated by turning around that coverslip so that the bottom and top parts of the coverslip were exchanged. In absence of this action, most of microspheres in the suspension move to the bottom of glass coverslip and aggregate into multilayered clusters. The decrease of edge angle and exchange between bottom and top parts of the coverslip causes a back-flow of the suspension which carries some of the microspheres back from bottom to disperse and fills the defects in the monolayer formed during the first spreading step.

To determine the optimum volume of aqueous solution needed to wet the slide, we initially spread a drop of water instead of suspension on the cleaned glass coverslip. Through measuring the spreading time of the front edge of water arriving at the bottom of coverslip, we found that a volume of 40 - $60 ¥$ is suitable for optimal wetting (Table SI1). $45 ¥$ is the volume used consistently throughout our experiments. This provides, on one hand enough time for particle absorption before draining, and on the other hand, leaves enough time before evaporation for inducing a back-flow as described above. 
We optimized the concentration of beads in the suspension for the optimal volume determined above. Dilution of 1:2, 1:4, 1:5 and 1:6 ( $\left.v_{\text {suspension }} / v_{\text {water }}\right)$ were carried out. In case of $2 \ddagger$ silica particles, when the dilution was less than 1:4, large areas of bead multi-layers were obtained. On the other hand, when the dilution was more than 1:6, lot of defects were observed. A dilution of 1:5 was found to be optimal and was chosen. The procedure was repeated for each type of bead to find the optimal spreading condition.

Fig. SI 1 shows typical examples of masks visualized by optical microscopy in transmitted light. 

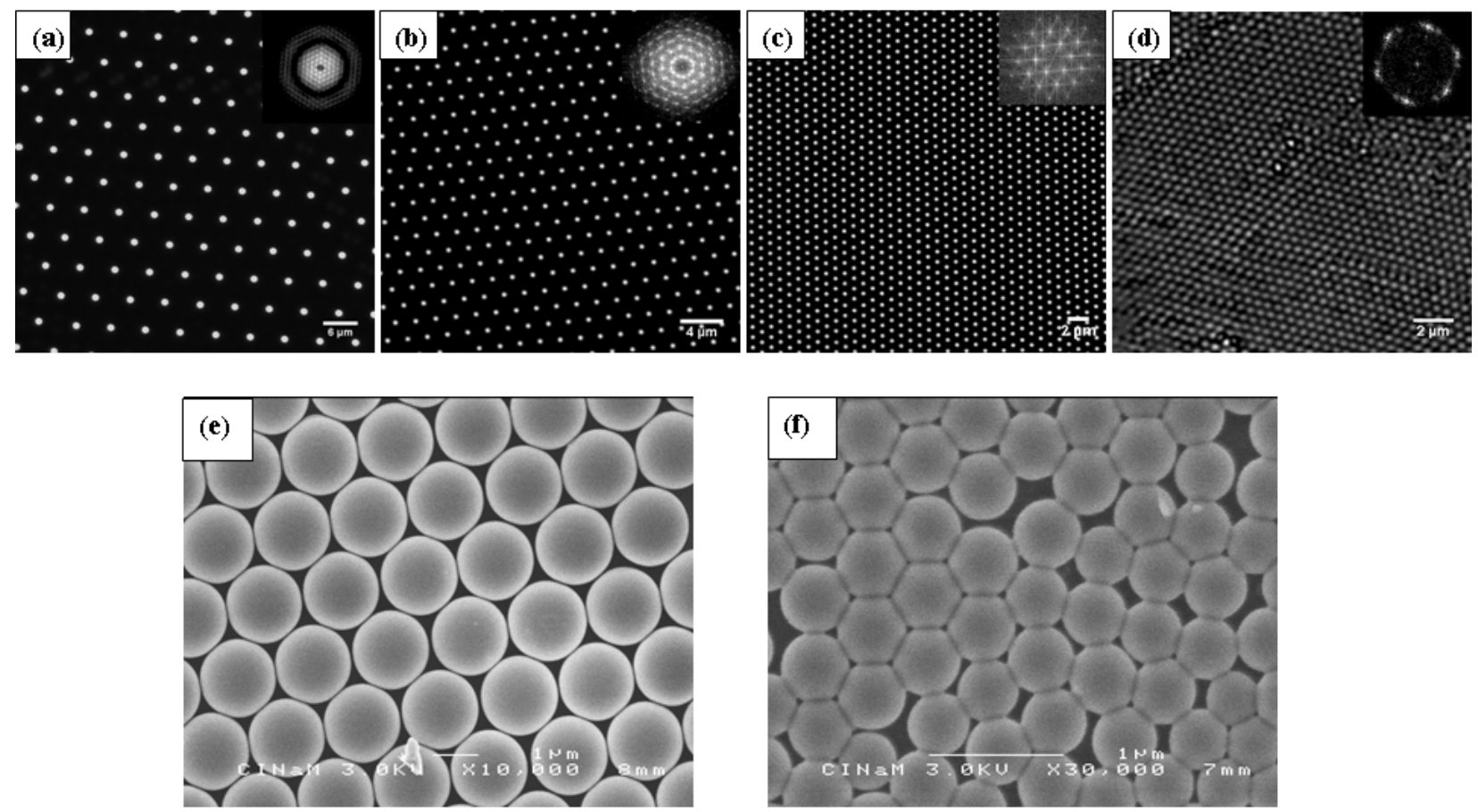

Figure SI 1. Top row: Optical microscopy (transmission bright field) images of colloidal bead masks on glass substrate (a) $6 \nsucceq$ PS beads (b) $2 ¥ n$ silica beads (c) $1 \nsucceq$ PS beads (d) 500 nm silica beads; Insets display the Fourier transforms of the corresponding images to emphasize the ordering of the lattice. The images were taken with a 100 x 1.4 NA oil immersion objective. Bottom row: Scanning electron microscopy images of the masks (e) $2 ¥ n$ silica beads (f) $500 \mathrm{~nm}$ silica beads (acquired with Joel JSM-6320F, Japan). A fine layer of carbon was deposited on the masks to render them conducting in order to prevent artifact arising from over charging during imaging.

Table SI 1: Spreading time of front edge of water arriving at the bottom of cleaned glass coverslip, $24{ }_{2}^{3} 24 \mathrm{~mm}$, with edge angle of $13^{\circ}$.

\begin{tabular}{lcccccc}
\hline $\begin{array}{l}\text { Volume } \\
\text { (water) }\end{array}$ & $20 ¥$ & $30 ¥$ & $40 ¥$ & $50 ¥$ & $60 ¥$ & $70 \sharp$ \\
\hline
\end{tabular}




\begin{tabular}{lllllll}
\hline $\begin{array}{l}\text { Spreading } \\
\text { time } \\
\text { (second) }\end{array}$ & 182 & 91 & 57 & 30 & 23 & 11 \\
\end{tabular}

\section{Silinization, grafting of PEG and protein patterning.}

Octadecyl trichlorosilane (OTS), (3-Aminopropyl) triethoxysilane (APTES), anhydrous $\mathrm{N}, \mathrm{N}$ Dimethyl formamide (DMF), Bovine serum albumin (BSA - either coupled to Texas-Red or to biotin), neutravidin (avidin analogue) covalently linked to the Texas Red, as well as all solvent and buffers (chloroform, 2-Propanol, phosphate buffered saline - PBS etc) were purchased from Sigma, France unless otherwise stated. N-Hydroxy succinimide-polyethylene glycol (NHS-PEG) ester (Mw: $509 \mathrm{Da}, 736 \mathrm{Da}, 2015 \mathrm{Da}$, with spacer arm length of $3.1 \mathrm{~nm}, 7.3 \mathrm{~nm}, 13.5 \mathrm{~nm}$, ) was purchased from RAPP Polymere.

Silanization with OTS or APTES was carried out under vacuum at $60^{\circ} \mathrm{C}$ for one hour in a closed vessel. To graft PEG to APTES, the silinized coverslip was immersed in NHS-PEG/DMF solution (50 of $250 \mathrm{mM}$ NHS-PEG/DMF added to $2 \mathrm{~mL}$ PBS buffer solution, $\mathrm{pH}=7.4$ ) at room temperature for 50 minutes. The PEG grafted coverslip was rinsed several times in ultrapure water and gently dried with nitrogen stream. The colloidal bead mask was then removed using a double sided sticky tape, or by sonication, either in water (for silica) or chloroform (for PS).

To further functionalize with protein, the PEG grafted coverslip, with the mask removed, was incubated in $300 ¥$ of $10 \neq / \mathrm{ml} \mathrm{BSA}$ in PBS. For proof-of-principle pilot experiments, fluorescent BSA was used whereas for tests with cells, BSA coupled to biotin was used. In the latter case, further incubation with 2 /ml Texas-Red coupled neutravidin in PBS buffer solution followed by incubation in mono-biotinylated anti-CD3 (OKT3 kind gift from Rajat Varma, NIH) was performed. Each incubation lasted 30 minutes at room temperature. Excess 
protein was removed by exchanging the buffer against protein free PBS in a series of typically ten washing steps.

\section{Preparation of PDMS substrates.}

Polydimethylsiloxane Sylgard 184 (PDMS) was purchased from Dow Corning, USA and Trichloro( $1 H, 1 H, 2 H, 2 H$-perfluorooctyl)silane (fluoro-silane) from Sigma, France. The PDMS elastomer base and the curing agent was mixed in the desired ratio - 10:1, 35:1 and 50:1 ( $w_{\text {base }} / w_{\text {agent }}$ ), to obtain hard, medium and soft PDMS respectively (see Ref. 31 and 32 of main text). The PDMS substrates were fabricated by curing the base/curing agent mixture between two glass coverslips, separated using glass spacers of about 140 micron, and baked at $60^{\circ} \mathrm{C}$ for 16 hours. One of the coverslips was destined to act as the support for the PDMS sheet. The other coverslip was silanized with fluoro-silane (from vapor phase in a closed desiccator under vacuum at room temperature for 30 minutes) prior to contact with uncured PDMS. After curing a drop of 2-propanol was injected into the gap between the coverslips to facilitate their separation. On separation, PDMS sheets of thickness corresponding to the spacers, supported on glass and with one smooth surface were obtained. The glass supported PDMS substrates were ultrasonicated in ultrapure water (3 times 10 minutes) with repeated flushing and blow-drying with nitrogen to eliminate the propanol. Finally, dried PDMS sheets were desiccated at $\sim 40 \mathrm{KPa}$ vacuum at room temperature overnight to remove the residual propanol on PDMS surface.

Protein nano-dot arrays on glass were prepared as described above, choosing the concentration of the fluorescent BSA solution for the final incubation as $20 \quad \mathrm{ml}$. After verification of the quality of the nano-dot array on the glass master by epi-fluorescence microscopy, the glass supported PDMS sheet prepared as above were put in contact with the glass master. Uniform pressure was applied manually to effectuate the transfer. The protein nano-dot pattern was readily 
transferred to the PDMS surface. The substrates were then imaged in epi-fluorescence to asses the quality of transfer.

\section{Imaging of patterns and data analysis.}

Epi-fluorescence images of the protein nano-dot arrays were acquired on an inverted optical microscope (Axio Observer, Zeiss, Germany) equipped with an EM-CCD camera (Andor, UK) and high magnification objective ( $100_{2}^{3}$, NA 1.4, Zeiss, Germany). Images were analyzed with Fiji [Nature Methods 9(7): 676-682]/ImageJ v1.47 (http://imagej.nih.gov/ij/) and IgorPro (WaveMatrics, USA) software packages. For the intensity traces presented (Fig. 3 and 5 of main text), the camera offset was subtracted from the data prior to analysis: no other modification was done to the raw data (no background subtraction, for example). The reported size of the dots correspond to half width at full maximum. Statistics over at least 3 samples and 10 dots are presented. The error-bars are standard deviations.

Tapping mode AFM measurements were carried out on a NTEGRA system (NT-MDT, Russia): in air after grafting APTES and in PBS buffer solution $(\mathrm{pH}=7.4)$, after depositing protein. Silicon tips (NSC35, MicroMash, Bulgaria) with a typically resonance frequency of 120 $\mathrm{kHz}$ and less than $10 \mathrm{~nm}$ tip radius were used. AFM images were analyzed using NTEGRA Imaging Analysis 2.1.2 software packages. Images were flattened following standard practice. Statistics over at least 10 dots are presented. The error-bars are standard deviations.

\section{Cell culture, marking and cell imaging.}

Jurkat E6 T-lymphocytes were cultivated in RPMI complete medium supplemented with $10 \%$ Foetal Bovine Serum and with 1\% GlutaMAX (Invitrogen). Last splitting was realized 24h before experiments to ensure a concentration of 0.6 million cells $/ \mathrm{mL}$ at the moment of the 
experiment. Cells were deposited on the substrates in Hepes-BSA buffer (20mM HEPES pH 7.2, $137 \mathrm{mM} \mathrm{NaCl}, 5 \mathrm{mM} \mathrm{KCl}, 0.7 \mathrm{mM} \mathrm{Na} \mathrm{HPO}_{4}, 6 \mathrm{mM}$ D-glucose, $2 \mathrm{mM} \mathrm{MgCl}$, $1 \mathrm{mM} \mathrm{CaCl}$, $0.025 \% \mathrm{BSA}$ ) and incubated during $15 \mathrm{~min}$ at $37^{\circ} \mathrm{C} 5 \% \mathrm{CO}_{2}$. Fixation was performed $15 \mathrm{~min}$ after deposition with $2 \%$ paraformaldehyde during $10 \mathrm{~min}$ at $37^{\circ} \mathrm{C}$ and followed by extensive rinsing with PBS. For fluorescent experiments, the cells were labeled by incubation with $1 \mu \mathrm{g} / \mathrm{ml}$ of Alexa 488 labeled antibody directed against the T-Cell receptor (anti Vbeta8, Ebiosciences) during $60 \mathrm{~min}$, followed by extensive rinsing.

Observation was performed on a Zeiss inverted microscope equipped with an Andor EMCCD camera. Reflection interference Contrast Microscopy (RICM) was performed with a 63x $\mathrm{NA}=1.25$ Antiflex oil immersion objective (Zeiss, Germany) and using $540 \mathrm{~nm}$ green line of a fluorescence excitation light source (X-cite, Canada) . Total Internal Reflection Fluorescence microscopy (TIRF-M) was performed using an Argon laser filtered at $488 \mathrm{~nm}$ in excitation using a 100x, NA=1.45 oil immersion objective (Zeiss, Germany). IRM (Interference Reflection Microscopy) was performed with the same objective using a mercury lamp illumination filtered at $546 \mathrm{~nm}$ and a semi-reflecting mirror. For observation through the PDMS layer (data not shown), a NA=1.40 oil immersion objective was used instead to exploit longer working distance. See Ref. 29 of main text for information on RICM and IRM.

\section{Alternative protocol for PEG grafting.}

In this protocol, the PEG molecular chain was covalently grafted on to glass surface through bonding of carboxyl group of PEG-COOH to amide group at the terminal of APTES (Fig. SI 2). By activating the amide group into activated ester with EDC (1-(3-Dimethylaminopropyl)-3ethylcarbodiimide hydrochloride), the amide group could readily bind to the carboxyl group in the presence of slight excess of NMM ( $N$-methylmorpholine). Since the activated ester is not stable, protective NHS ( $N$-Hydroxysuccinimide) was added into the reactive system to extend 
the life of activated ester and allow for full grafting APTES. All reagents were from Sigma, France.
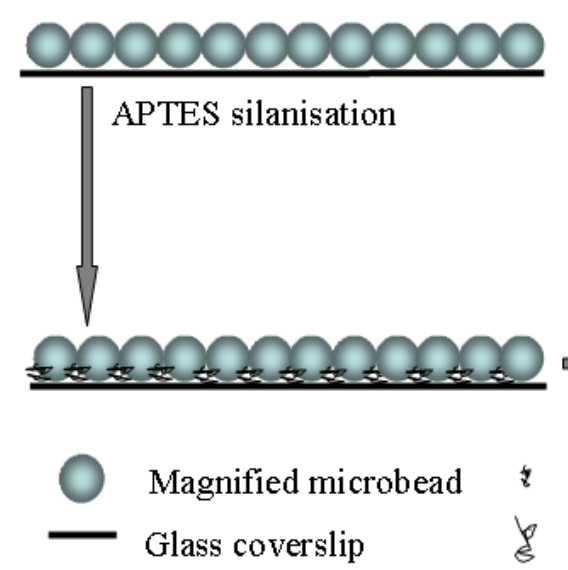

APTES

\& APTES bonded PEG

Figure SI 2. Schematic representation of the strategy used to bind PEG-COOH molecules to patterned APTES. 